\title{
SMALL ZEROS OF QUADRATIC FORMS OVER NUMBER FIELDS
}

\author{
JEFFREY D. VAALER
}

\begin{abstract}
Let $F$ be a nontrivial quadratic form in $N$ variables with coefficients in a number field $k$ and let $A$ be a $K \times N$ matrix over $k$. We show that if the simultaneous equations $F(\mathbf{x})=0$ and $A \mathbf{x}=0$ hold on a subspace $\chi$ of dimension $L$ and $L$ is maximal, then such a subspace $X$ can be found with the height of $X$ relatively small. In particular, the height of $X$ can be explicitly bounded by an expression depending on the height of $F$ and the height of $A$. We use methods from geometry of numbers over adele spaces and local to global techniques which generalize recent work of H. P. Schlickewei.
\end{abstract}

1. Introduction. Let

$$
F(\mathbf{x}, \mathbf{y})=\sum_{m=1}^{N} \sum_{n=1}^{N} \varphi_{m n} y_{m} x_{n}
$$

be a symmetric bilinear form with coefficients $\varphi_{m n}=\varphi_{n m}$ in an algebraic number field $k$. We write $\Phi=\left(\varphi_{m n}\right)$ for the associated $N \times N$ matrix and $F(\mathbf{x})=F(\mathbf{x}, \mathbf{x})$ for the associated quadratic form. It is well known that if the quadratic form $F$ has a nontrivial zero in $k^{N}$ then in fact there is a vector $\varsigma \neq 0$ in $k^{N}$ such that $F(\varsigma)=0$ and

$$
h(\varsigma) \ll_{k, N} H(\Phi)^{(N-1) / 2} .
$$

Here $h$ is a homogeneous height on vectors and $\mathcal{H}$ is a suitable homogeneous height on the matrix $\Phi$. We give precise definitions for all our heights in $\S 2$. The bound (1.2) was obtained for $k=\mathbf{Q}$ by Cassels [3] (see also the version of Birch and Davenport [1]), and later generalized to number fields by Chalk [4] and S. Raghavan [7]. Recently H. P. Schlickewei [8] has extended Cassels' result for $k=\mathbf{Q}$ in a different direction. Suppose that $L \geq 1$ is the largest integer such that the quadratic form $F$ vanishes on some $L$ dimensional rational subspace of $\mathbf{Q}^{N}$. Then Schlickewei has shown that there exist $L$ linearly independent vectors $\varsigma_{1}, \varsigma_{2}, \ldots, \varsigma_{L}$ in $\mathbf{Q}^{N}$ such that $F$ vanishes identically on the subspace spanned by $\left\{\varsigma_{1}, \varsigma_{2}, \ldots, \varsigma_{L}\right\}$, and

$$
\prod_{l=1}^{L} h\left(\varsigma_{l}\right) \ll_{N} H(\Phi)^{(N-L) / 2} .
$$

In particular, there exists a vector $\varsigma_{1} \neq 0$ in $\mathbf{Q}^{N}$ with $F\left(\varsigma_{1}\right)=0$ and

$$
h\left(\varsigma_{1}\right) \ll_{N} \mathcal{H}(\Phi)^{(N-L) / 2 L} .
$$

Received by the editors July 4, 1986.

1980 Mathematics Subject Classification (1985 Revision). Primary 11D09; Secondary 11 H55.

Research supported by a grant from the National Science Foundation, DMS-8501941. 
In the present paper we further extend Schlickewei's result by working over an arbitrary algebraic number field $k$. This requires us to use geometry of numbers over adèle spaces. We also consider simultaneous zeros of a quadratic form $F$ and a system of linear forms $A \mathbf{x}$, where $A$ is a $K \times N$ matrix over $k$ with rank $(A)=K<N$. Our bounds depend on $H(\Phi)$ and on the height $H(A)$ of the system of linear forms. Here $H(A)$ is the homogeneous height on the Grassmann coordinates of $A$ which occurred in [2].

2. Heights and measures. We suppose that the number field $k$ has degree $d$ over $\mathbf{Q}$. If $v$ is a place of $k$ we write $k_{v}$ for the completion of $k$ at $v$. Then $d_{v}=\left[k_{v}: \mathbf{Q}_{v}\right]$ denotes the local degree. We write $v \mid \infty$ if $v$ is an infinite place, $v \nmid \infty$ if $v$ is a finite place, and $v \mid p$ if $v$ lies over the prime number $p$. At each place $v$ we normalize an absolute value ||$_{v}$ on $k_{v}$ as follows:

(i) if $v \mid p$ then $|p|_{v}=p^{-d_{v} / d}$,

(ii) if $v \mid \infty$ then $|\alpha|_{v}=|\alpha|^{d_{v} / d}$, where || is the usual Euclidean absolute value on $\mathbf{R}$ or $\mathbf{C}$.

It follows that our absolute values satisfy the product formula $\prod_{v}|\alpha|_{v}=1$ whenever $\alpha \in k$ and $\alpha \neq 0$. We will also make use of a second normalized absolute value \|\|$_{v}$ on $k_{v}$ which we define by

$$
\|\alpha\|_{v}=|\alpha|_{v}^{d / d v} .
$$

Of course \|\|$_{v}$ extends the usual $p$-adic absolute value on $\mathbf{Q}_{p}$ if $v \mid p$ and the Euclidean absolute value if $v / \infty$.

Let

$$
\mathbf{x}=\left(\begin{array}{c}
x_{1} \\
x_{2} \\
\vdots \\
x_{N}
\end{array}\right)
$$

denote a (column) vector in $k^{N}$. We define a height $h(\mathbf{x})$ for such vectors by

$$
h(\mathbf{x})=\prod_{v}\left\{\max _{1 \leq n \leq N}\left|x_{n}\right|_{v}\right\} .
$$

If $\alpha \neq 0$ and $\alpha \in k$, then by the product formula we have $h(\alpha \mathbf{x})=h(\mathbf{x})$. This homogeneity allows us to regard $h(\mathbf{x})$ as an absolute height on projective space $\mathbf{P}_{k}^{N-1}$.

It will be convenient to extend our absolute value \|\|$_{v}$ to vectors $\mathbf{x}$ in $\left(k_{v}\right)^{N}$ as follows:

(i) if $v \nmid \infty$ then $\|\mathbf{x}\|_{v}=\max _{1 \leq n \leq N}\left\|x_{n}\right\|_{v}$,

(ii) if $v \mid \infty$ then $\|\mathbf{x}\|_{v}=\left(\sum_{n=1}^{N}\left\|x_{n}\right\|_{v}^{2}\right)^{1 / 2}$.

We also extend ||$_{v}$ to vectors by $\|\mathbf{x}\|_{v}^{d_{v} / d}=|\mathbf{x}|_{v}$.

Next we consider heights on matrices. If $\Phi=\left(\varphi_{m n}\right)$ is an $N \times N$ matrix over $k$ we set

$$
\begin{gathered}
\mathcal{H}_{v}(\Phi)=\max _{1 \leq m, n \leq N}\left|\varphi_{m n}\right|_{v} \quad \text { if } v \nmid \infty, \\
\mathcal{H}_{v}(\Phi)=\left\{\sum_{m=1}^{N} \sum_{n=1}^{N}\left\|\varphi_{m n}\right\|_{v}^{2}\right\}^{d_{v} / 2 d} \quad \text { if } v \mid \infty,
\end{gathered}
$$


and

$$
\mathcal{H}(\Phi)=\prod_{v} H_{v}(\Phi)
$$

If $\alpha \neq 0$ and $\alpha \in k$ then we clearly have $\mathcal{H}(\alpha \Phi)=\mathcal{H}(\Phi)$ by the product formula. We will apply the height $\nLeftarrow$ only to the square matrix $\Phi$ of coefficients for the quadratic form $F$.

Let $X=\left(x_{n m}\right)$ be an $N \times M$ matrix over $k_{v}$. If $I \subseteq\{1,2, \ldots, N\}$ is a subset of cardinality $|I|=L$, we write

$$
{ }_{I} X=\left(x_{n m}\right), \quad n \in I, m=1,2, \ldots, M,
$$

for the corresponding $L \times M$ submatrix. Similarly, if $J \subseteq\{1,2, \ldots, M\}$ with $|J|=L$, we write

$$
X_{J}=\left(x_{n m}\right), \quad n=1,2, \ldots, N, m \in J,
$$

for the corresponding $N \times L$ submatrix. Now suppose that $\operatorname{rank}(X)=M \leq N$. We define the local height $H_{v}(X)$ on the Grassmann coordinates of $X$ as follow:

(i) If $v \nmid \infty$ then

$$
H_{v}(X)=\max _{|I|=M}\left|\operatorname{det}_{I} X\right|_{v}
$$

(ii) If $v \mid \infty$ then

$$
H_{v}(X)=\left(\sum_{|I|=M}\left\|\operatorname{det}_{I} X\right\|_{v}^{2}\right)^{d_{v} / 2 d}
$$

We note that for $v \mid \infty$ we also have

$$
H_{v}(X)=\left|\operatorname{det} X^{*} X\right|_{v}^{1 / 2}
$$

by the Cauchy-Binet formula, where $X^{*}$ denotes the complex conjugate transpose of $X$. The identity (2.2) will be used frequently.

The local heights $H_{v}$ satisfy an important inequality. Suppose that $\operatorname{rank}(X)=$ $M \leq N$ with $X$ partitioned into blocks as $X=\left(X_{1} X_{2}\right)$, where $X_{1}$ is $N \times M_{1}$ and $X_{2}$ is $N \times M_{2}$. Then we have

$$
H_{v}(X) \leq H_{v}\left(X_{1}\right) H_{v}\left(X_{2}\right) .
$$

This is easily proved using Laplace's expansion. Further details are contained in [2, pp. 15-16].

If $\mathbf{x}$ is a (column) vector in $\left(k_{v}\right)^{N}$ we write $H_{v}(\mathbf{x})$ for the local height of $\mathbf{x}$ where we regard $\mathrm{x}$ as an $N \times 1$ matrix. We note the identity

$$
H_{v}(\mathbf{x})=\left(\|\mathbf{x}\|_{v}\right)^{d_{v} / d}=|\mathbf{x}|_{v} .
$$

If $X=\left(x_{n m}\right)$ has entries in $k$ we may apply $H_{v}$ at each place $v$ of $k$. In this case we define the global height $H(X)$ by

$$
H(X)=\prod_{v} H_{v}(X) .
$$


Thus $H$ is a height on the vector $\left(\operatorname{det}_{I} X\right),|I|=M$, of Grassmann coordinates of $X$. Suppose that $U$ is an $M \times M$ nonsingular matrix over $k$. Then

$$
H(X U)=\prod_{v}\left\{|\operatorname{det} U|_{v} H_{v}(X)\right\}=H(X)
$$

by the product formula. This identity allows us to view $H$ as a height on subspaces.

Let $\chi \subseteq k^{N}$ be a vector subspace of dimension $M, 1 \leq M \leq N$, and let $\left\{\mathbf{x}_{1}, \mathbf{x}_{2}, \ldots, \mathbf{x}_{M}\right\}$ be a basis for $\chi$ over $k$. We may then suppose that

$$
X=\left(\mathbf{x}_{1} \mathbf{x}_{2} \cdots \mathbf{x}_{M}\right)
$$

is the $N \times M$ matrix having $\mathbf{x}_{m}$ as its $m$ th column. Assume that $Y=\left(\mathbf{y}_{1} \mathbf{y}_{2} \cdots \mathbf{y}_{M}\right)$ is another $N \times M$ matrix having columns which form a basis for $\chi$ over $k$. Then there exists an $M \times M$ nonsingular matrix $U$ such that $Y=X U$. In view of $(2.4)$ we have $H(Y)=H(X)$. Hence we define the height $H(\mathcal{X})$ of the subspace $\mathcal{X}$ by

$$
H(\chi)=H(X) \text {. }
$$

Our remarks show that this is well defined.

Let $X=\left(x_{n m}\right)$ be an $N \times M$ matrix over $k_{v}$ or $k$ with $\operatorname{rank}(X)=N \leq M$. We extend our heights $H_{v}$ and $H$ to $X$ by applying them to the transpose of $X$.

The subspace $\chi \subseteq k^{N}$ may occur as the null space of a system of linear forms. Let $A$ be an $(N-\bar{M}) \times N$ matrix over $k$ with $\operatorname{rank}(A)=N-M<N$ and such that $X=\left\{\mathbf{y} \in k^{N}: A \mathbf{y}=0\right\}$. By the duality theorem of Brill-Gordan [5] (see also [6, Theorem I, p. 294]) there exists a constant $\gamma \in k, \gamma \neq 0$, such that for every subset $I \subseteq\{1,2, \ldots, N\},|I|=M$,

$$
\operatorname{det}_{I} X=\gamma(-1)^{\varepsilon(J)} \operatorname{det} A_{J},
$$

where $J$ is the complement of $I$ and $\varepsilon(J)=\sum_{j \in J} j$. From the product formula we therefore obtain the following duality principle:

$$
H(X)=\prod_{v} H_{v}(X)=\prod_{v}\left\{|\gamma|_{v} H_{v}(A)\right\}=H(A) .
$$

A basic property of our heights $h, \forall$ and $H$ is that they depend only on the algebraic numbers which form the entries or coordinates of the matrices and vectors to which these heights are applied. They do not depend on the field $k$ which contains the entries or coordinates. This follows from the way we have normalized the absolute values ||$_{v}$.

In $\S 5$ we apply an adèlic version of Minkowski's second theorem on successive minima. This result and the relevant definitions concerning geometry of numbers over adèle spaces are contained in [2, pp. 16-18]. Here we briefly describe some of the objects which occur in our application of Minkowski's theorem.

We write $k_{\mathbf{A}}$ for the adèle ring of the number field $k$, and $O_{k}$ for the ring of integers of $k$. If $v$ is a finite place of $k$ then

$$
O_{v}=\left\{x \in k_{v}:|x|_{v} \leq 1\right\}
$$

denotes the maximal compact subring of $k_{v}$. Now the additive group of $k_{v}$ is locally compact. Therefore we may select a Haar measure $\beta_{v}$ on $k_{v}$ by the following normalization: 
(i) If $v \mid p$ we require that $\beta_{v}\left(O_{v}\right)=\left|D_{v}\right|_{v}^{d / 2}$, where $D_{v}$ is the local different of $k$ at $v$.

(ii) If $k_{v}=\mathbf{R}$ then $\beta_{v}$ is ordinary Lebesgue measure on $\mathbf{R}$.

(iii) If $k_{v}=\mathbf{C}$ then $\beta_{v}$ is Lebesgue measure on the complex plane multiplied by 2 .

The product measure $\beta=\prod_{v} \beta_{v}$ then induces a normalized Haar measure (also denoted by $\beta$ ) on the adèle ring $k_{\mathbf{A}}$. If $\left(k_{\mathbf{A}}\right)^{N}$ is an $N$-fold product of adèle spaces we write $V$ for the product Haar measure $\beta^{N}$ on $\left(k_{\mathbf{A}}\right)^{N}$. In the adèlic version of Minkowski's second theorem the Haar measure $V$ plays the rôle of volume.

It will be convenient to define

$$
r_{v}(N)= \begin{cases}\pi^{-1 / 2}\left\{\Gamma\left(\frac{1}{2} N+1\right)\right\}^{1 / N} & \text { if } v \text { is real } \\ (2 \pi)^{-1 / 2}\{\Gamma(N+1)\}^{1 / 2 N} & \text { if } v \text { is complex } \\ 1 & \text { if } v \nmid \infty,\end{cases}
$$

and

$$
c_{k}(N)=\left\{2\left|\Delta_{k}\right|^{1 / 2 d} \prod_{v \mid \infty}\left(r_{v}(N)\right)^{d_{v} / d}\right\},
$$

where $\Delta_{k}$ is the discriminant of $k$. The radii $r_{v}(N)$ and our normalization of $\beta_{v}$ are such that

$$
\beta_{v}^{N}\left(\left\{\mathbf{u} \in\left(k_{v}\right)^{N}:\|\mathbf{u}\|_{v}<r_{v}(N)\right\}\right)=1
$$

for each infinite place $v$ of $k$. The quantity $c_{k}(N)$ will occur as a field constant.

3. Statement of results. Let $F(\mathbf{x}, \mathbf{y})$ be a symmetric bilinear form over $k$ with $N \times N$ coefficient matrix $\Phi$ as in (1.1). Let $Z \subseteq k^{N}$ be a subspace of dimension $M$, $1 \leq M \leq N$. We assume that the quadratic form $F(\mathbf{x})=F(\mathbf{x}, \mathbf{x})$ has a nontrivial zero in $Z$ and we define $L$ to be the largest positive integer such that

$$
Z^{(0)}=\{\mathbf{z} \in Z: F(\mathbf{z})=0\}
$$

contains a subspace of dimension $L$. Thus we have $1 \leq L \leq M$, but to avoid degenerate situations we will assume that $1 \leq L<M$.

THEOREM 1. There exists a subspace $\mathcal{X} \subseteq Z^{(0)}$ such that $\mathcal{X}$ has dimension $L$ over $k$ and

$$
H(X) \leq\left\{2^{2 L+1} c_{k}(M-L)^{2} \nLeftarrow(\Phi)\right\}^{(M-L) / 2} H(Z) .
$$

By using a basis for $\mathcal{X}$ in Theorem 1 consisting of vectors with small height, we obtain the following generalization of Satz 1 in Schlickewei [8].

COROLlaRY 2. There exist $L$ linearly independent vectors $\varsigma_{1}, \varsigma_{2}, \ldots, \varsigma_{L}$ in $\left(O_{k}\right)^{N} \cap Z^{(0)}$ such that $F$ vanishes identically on the subspace $X$ spanned by $\left\{\varsigma_{1}, \varsigma_{2}\right.$, $\left.\ldots, \varsigma_{L}\right\}$ over $k$ and such that

$$
\prod_{l=1}^{L} h\left(\varsigma_{l}\right) \leq c_{k}(1)^{L}\left\{2^{2 L+1} c_{k}(M-L)^{2} \nLeftarrow(\Phi)\right\}^{(M-L) / 2} H(Z) .
$$

If the vectors $\varsigma_{1}, \varsigma_{2}, \ldots, \varsigma_{L}$ in Corollary 2 are arranged in order of increasing height then it is clear that $h\left(\varsigma_{1}\right)$ satisfies an inequality analogous to (1.4). 
Let $A$ be a $K \times N$ matrix over $k$ with $\operatorname{rank}(A)=K<N$. Assume that

$$
Z^{(0)}=\left\{\mathbf{z} \in k^{N}: A \mathbf{z}=0 \text { and } F(\mathbf{z})=0\right\}
$$

contains a nontrivial vector and then let $L$ be the largest positive integer such that $Z^{(0)}$ contains a subspace of dimension $L$. Now to avoid degeneracies we suppose that $K+L<N$.

COROLlaRY 3. There exists a subspace $\chi$ contained in the set $Z^{(0)}$ defined by (3.3) such that $\mathcal{X}$ has dimension $L$ over $k$ and

$$
H(X) \leq\left\{2^{2 L+1} c_{k}(N-K-L) \nLeftarrow(\Phi)\right\}^{(N-K-L) / 2} H(A) .
$$

Moreover, there exist $L$ linearly independent vectors $\varsigma_{1}, \varsigma_{2}, \ldots, \varsigma_{L}$ in $\left(O_{k}\right)^{N} \cap Z^{(0)}$ such that $\chi$ is spanned by $\left\{\zeta_{1}, \zeta_{2}, \ldots, \zeta_{L}\right\}$ over $k$ and

$$
\prod_{l=1}^{L} h\left(\varsigma_{l}\right) \leq c_{k}(1)^{L}\left\{2^{2 L+1} c_{k}(N-K-L) \nLeftarrow(\Phi)\right\}^{(N-K-L) / 2} H(A) .
$$

The bounds given in (3.2) and (3.5) may be regarded as a hybrid version of Schlickewei's result proved over an algebraic number field and the homogeneous form of Siegel's lemma obtained in [2]. For example, if $Z=k^{N}$ then (3.2) reduces to (1.3). On the other hand, in the degenerate situation $K+L=N$ the inequality (3.5) becomes

$$
\prod_{l=1}^{L} h\left(\varsigma_{l}\right) \leq c_{k}(1)^{L} H(A)
$$

and this is exactly the bound given in [2, Theorem 9].

4. Preliminary lemmas. Throughout this section we work over the vector space $\left(k_{v}\right)^{N}$, where $v$ is a fixed place of $k$. Let $A \subseteq\left(k_{v}\right)^{N}$ be a subspace of dimension $L, 1 \leq L \leq N$, and suppose that $\left\{\mathbf{a}_{1}, \mathbf{a}_{2}, \ldots, \mathbf{a}_{L}\right\}$ is a basis for $A$ as a vectorspace over $k_{v}$. We write $A=\left(\mathbf{a}_{1} \mathbf{a}_{2} \cdots \mathbf{a}_{L}\right)$ for the $N \times L$ matrix having $\mathbf{a}_{l}$ as its $l$ th column. Next we define an $N \times N$ matrix $P_{v}=P_{v}(A)$ as follows:

(i) If $v \mid \infty$ then

$$
P_{v}=A\left(A^{*} A\right)^{-1} A^{*},
$$

(ii) if $v \nmid \infty$ we select $J=J_{v} \subseteq\{1,2,3, \ldots, N\}$ so that $|J|=L$ and

$$
\max _{|I|=L}\left|\operatorname{det}_{I} A\right|_{v}=\left|\operatorname{det}{ }_{J} A\right|_{v} \text {. }
$$

Then we define

$$
P_{v}=A\left({ }_{J} A\right)^{-1}{ }_{J}\left(1_{N}\right)
$$

Here $1_{N}$ denotes the $N \times N$ identity matrix. If $\left\{\mathbf{a}_{1}^{\prime}, \mathbf{a}_{2}^{\prime}, \ldots, \mathbf{a}_{L}^{\prime}\right\}$ is another basis for $A$ and $A^{\prime}=\left(\mathbf{a}_{1}^{\prime} \mathbf{a}_{2}^{\prime} \cdots \mathbf{a}_{L}^{\prime}\right)$ is the corresponding $N \times L$ matrix, then $A=A^{\prime} U$, where $U$ is a nonsingular $L \times L$ matrix over $k_{v}$. A simple calculation shows that $P_{v}(A)=P_{v}\left(A^{\prime}\right)$. Thus we may write $P_{v}=P_{v}(A)$, since $P_{v}$ depends on the subspace $A$ and not on our choice of a basis for $A$.

The matrix $P_{v}$ acts as a projection operator onto the subspace $A$. In particular we have

$$
P_{v} \mathrm{x} \in \mathcal{A} \text { for all } \mathrm{x} \in\left(k_{v}\right)^{N}
$$


and

$$
P_{v} \mathbf{x}=\mathbf{x} \text { for all } \mathbf{x} \in A \text {. }
$$

The first of these is trivial. To verify (4.5), write $\mathbf{x} \in A$ as $\mathbf{x}=A \mathbf{y}$ with $\mathbf{y} \in\left(k_{v}\right)^{L}$. If $v \mid \infty$ we have

$$
P_{v} \mathbf{x}=A\left(A^{*} A\right)^{-1} A^{*} A \mathbf{y}=A \mathbf{y}=\mathbf{x}
$$

and if $v \nmid \infty$ then

$$
P_{v} \mathbf{x}=A\left({ }_{J} A\right)^{-1}{ }_{J}\left(1_{N}\right) A \mathbf{y}=A\left({ }_{J} A\right)^{-1}\left({ }_{J} A\right) \mathbf{y}=A \mathbf{y}=\mathbf{x} .
$$

Using (4.4) and (4.5), or by direct calculation, we also find that

$$
P_{v}^{2}=P_{v} .
$$

Let $M$ be an integer with $L<M \leq N$ and let $B=\left(\mathbf{b}_{1} \mathbf{b}_{2} \cdots \mathbf{b}_{M-L}\right)$ be an $N \times(M-L)$ matrix over $k_{v}$. We assemble the matrices $A$ and $B$ into blocks of an $N \times M$ matrix $C$ by defining

$$
C=\left(\begin{array}{ll}
A & B
\end{array}\right) \text {. }
$$

LEMMA 4. If $\operatorname{rank}(C)=M$ and $P_{v}=P_{v}(\mathcal{A})$ is projection onto the subspace A spanned by the columns of $A$, then

$$
H_{v}(C)=H_{v}(A) H_{v}\left(\left(1_{N}-P_{v}\right) B\right) .
$$

PROOF. First we assume that $v \mid \infty$. Then

$$
C^{*} C=\left(\begin{array}{cc}
A^{*} A & A^{*} B \\
B^{*} A & B^{*} B
\end{array}\right)
$$

and the matrix

$$
\left(\begin{array}{cc}
1_{L} & -\left(A^{*} A\right)^{-1} A^{*} B \\
0 & 1_{M-L}
\end{array}\right)
$$

has determinant one. If we multiply $C^{*} C$ on the right by (4.9) we find that

$$
\begin{aligned}
\operatorname{det}\left(C^{*} C\right) & =\operatorname{det}\left(\begin{array}{cc}
A^{*} A & 0 \\
B^{*} A & B^{*}\left(1_{N}-P_{v}\right) B
\end{array}\right) \\
& =\operatorname{det}\left(A^{*} A\right) \operatorname{det}\left\{B^{*}\left(1_{N}-P_{v}\right) B\right\} .
\end{aligned}
$$

Next we use the identity

$$
B^{*}\left(1_{N}-P_{v}\right) B=\left\{\left(1_{N}-P_{v}\right) B\right\}^{*}\left\{\left(1_{N}-P_{v}\right) B\right\},
$$

which follows from (4.6), and then apply $\|_{v}$ to both sides of (4.10). This proves the lemma when $v$ is an infinite place.

Now suppose that $v \nmid \infty$ and let $I \subseteq\{1,2, \ldots, N\}$ with $|I|=M$. From the definition of $P_{v}$ in this case it is clear that each column of ${ }_{I}\left(P_{v} B\right)$ is a linear combination of columns of ${ }_{I} A$. Thus we have

$$
\begin{aligned}
& \operatorname{det}{ }_{I} C=\operatorname{det}\left\{\left({ }_{I} A \quad{ }_{I} B\right)\right\}=\operatorname{det}\left\{\left({ }_{I} A{ }_{I} B-{ }_{I}\left(P_{v} B\right)\right)\right\} \\
& =\operatorname{det}\left\{\left({ }_{I} A{ }_{I}\left(1_{N}-P_{v}\right) B\right)\right\} \text {. }
\end{aligned}
$$

Using (2.3), it follows that

$$
H_{v}(C)=H_{v}\left\{\left(A \quad\left(1_{n}-P_{v}\right) B\right)\right\} \leq H_{v}(A) H_{v}\left\{\left(1_{N}-P_{v}\right) B\right\} .
$$


To establish the inequality in the opposite direction we argue as follows. Let

$$
I_{1} \subseteq\{1,2, \ldots, N\}, \quad \text { with }\left|I_{1}\right|=L,
$$

and

$$
I_{2} \subseteq\{1,2, \ldots, N\}, \quad \text { with }\left|I_{2}\right|=M-L .
$$

We assume that ${ }_{I_{1}} A$ is not singular. Then the matrix

$$
\left(\begin{array}{cc}
1_{L} & -\left({ }_{I_{1}} A\right)^{-1}{ }_{I_{1}} B \\
0 & 1_{M-L}
\end{array}\right)
$$

has determinant one. Multiplying on the right by (4.11), we obtain that

$$
\begin{aligned}
\operatorname{det}\left(\begin{array}{cc}
I_{1} A & I_{1} B \\
I_{2} A & I_{2} B
\end{array}\right) & =\operatorname{det}\left(\begin{array}{cc}
I_{1} A & 0 \\
I_{2} A & I_{2} B-{ }_{I_{2}} A\left(I_{1} A\right)^{-1}{ }_{I_{1}} B
\end{array}\right) \\
& =\operatorname{det}\left(I_{1} A\right) \operatorname{det}\left\{I_{2} B-I_{2} A\left(I_{1} A\right)^{-1}{ }_{I_{1}} B\right\} \\
& =\operatorname{det}\left(I_{1} A\right) \operatorname{det}\left\{I_{2}\left(1_{N}-A\left(I_{1} A\right)^{-1}{ }_{I_{1}}\left(1_{N}\right)\right) B\right\} .
\end{aligned}
$$

If we select $I_{1}=J_{v}$ so as to maximize $\left|\operatorname{det}\left(I_{1} A\right)\right|_{v}$ then

$$
H_{v}(A)\left|\operatorname{det}\left\{I_{2}\left(1_{N}-P_{v}\right) B\right\}\right|_{v}=\left|\operatorname{det}\left(\begin{array}{cc}
J_{v} A & J_{v} B \\
I_{2} A & I_{2} B
\end{array}\right)\right|_{v} \leq H_{v}(C) .
$$

Finally, we select $I_{2}$ so as to maximize the left-hand side of (4.12). Thus we have

$$
H_{v}(A) H_{v}\left\{\left(1_{N}-P_{v}\right) B\right\} \leq H_{v}(C),
$$

which completes the proof of the lemma.

Lemma 5. Let $A, B, C$ and $P_{v}=P_{v}(\mathcal{A})$ be as in Lemma 4. We assume that $\Psi$ is an $(M-L) \times N$ matrix over $k_{v}$ such that

(i) $\Psi A$ is an $(M-L) \times L$ zero matrix,

(ii) $\Psi B$ is an $(M-L) \times(M-L)$ nonsingular matrix.

Then we have

$$
|\operatorname{det} \Psi B|_{v} H_{v}(A) \leq H_{v}(\Psi) H_{v}(C) .
$$

PROOF. From (i) and the Cauchy-Binet formula we obtain the expansion

$$
\operatorname{det} \Psi B=\operatorname{det}\left\{\Psi\left(1_{N}-P_{v}\right) B\right\}=\sum_{|I|=M-L}\left(\operatorname{det} \Psi_{I}\right)\left(\operatorname{det}\left\{{ }_{I}\left(1_{N}-P_{v}\right) B\right\}\right) .
$$

If $v \mid \infty$ we apply the Cauchy-Schwarz inequality to the right-hand side of (4.14). If $v \nmid \infty$ we apply the ultrametric inequality. In either case we deduce that

$$
|\operatorname{det} \Psi B|_{v} \leq H_{v}(\Psi) H_{v}\left\{\left(1_{N}-P_{v}\right) B\right\} .
$$

Next we multiply both sides of $(4.15)$ by $H_{v}(A)$ and use the identity (4.8). In this way we obtain exactly the inequality of the lemma.

LEMMA 6. Let $A \subseteq\left(k_{v}\right)^{N}$ be a subspace of dimension $L, 1 \leq L \leq N$, and let $\mathbf{a}_{1} \neq 0$ be a vector in $A$. There exist vectors $\mathbf{a}_{2}, \mathbf{a}_{3}, \ldots, \mathbf{a}_{L}$ in $A$ such that

(i) the set $\left\{\mathbf{a}_{1}, \mathbf{a}_{2}, \ldots, \mathbf{a}_{L}\right\}$ is a basis for $A$ over $k_{v}$,

(ii) the $N \times L$ matrix $A=\left(\mathbf{a}_{1} \mathbf{a}_{2} \cdots \mathbf{a}_{L}\right)$ satisfies the identity

$$
H_{v}(A)=\prod_{\ell=1}^{L} H_{v}\left(\mathbf{a}_{\ell}\right)
$$


ProOF. If $L=1$ the lemma is trivial. Therefore we assume that the lemma holds for all subspaces of dimension $L-1$, with $2 \leq L \leq N$. Given $\mathbf{a}_{1}$, let $\left\{\mathbf{a}_{1}, \mathbf{b}_{1}, \mathbf{b}_{2}, \ldots, \mathbf{b}_{L-1}\right\}$ be any basis for $A$ with $B=\left(\mathbf{b}_{1} \mathbf{b}_{2} \cdots \mathbf{b}_{L-1}\right)$. Then write $P_{v}=P_{v}\left(\mathbf{a}_{1}\right)$ for projection onto the subspace spanned by $\mathbf{a}_{1}$. By Lemma 4 we have

$$
H_{v}\left\{\left(\begin{array}{ll}
\mathbf{a}_{1} & B
\end{array}\right)\right\}=H_{v}\left(\mathbf{a}_{1}\right) H_{v}\left\{\left(1_{N}-P_{v}\right) B\right\} .
$$

Let $B^{\prime}$ be the subspace of $A$ spanned by $\left\{\left(1_{N}-P_{v}\right) \mathbf{b}_{1}, \ldots,\left(1_{N}-P_{v}\right) \mathbf{b}_{L-1}\right\}$. In view of (4.16), the subspace $B^{\prime}$ has dimension $L-1$. Applying the inductive hypothesis to $B^{\prime}$, we find that there exists a nonsingular, $(L-1) \times(L-1)$ matrix $U$ such that

$$
\left(1_{N}-P_{v}\right) B U=\left(\mathbf{b}_{1}^{\prime} \mathbf{b}_{2}^{\prime} \cdots \mathbf{b}_{L-1}^{\prime}\right)=B^{\prime}
$$

satisfies

$$
H_{v}\left(B^{\prime}\right)=\prod_{\ell-1}^{L-1} H_{v}\left(\mathbf{b}_{\ell}^{\prime}\right) .
$$

For $l=2,3, \ldots, L$ we define $\mathbf{a}_{l}=\mathbf{b}_{l-1}$. Then we have

$$
\begin{aligned}
H_{v}(A) & =H_{v}\left\{\left(\mathbf{a}_{1} \quad\left(1_{N}-P_{v}\right) B U\right)\right\} \\
& =H_{v}\left(\mathbf{a}_{1}\right) H_{v}\left\{\left(1_{N}-P_{v}\right) B U\right\}=H_{v}\left(\mathbf{a}_{1}\right) H_{v}\left(B^{\prime}\right)=\prod_{l=1}^{L} H_{v}\left(\mathbf{a}_{l}\right),
\end{aligned}
$$

which proves the lemma.

LEMMA 7. Let $A \subseteq\left(k_{v}\right)^{N}$ be a subspace of dimension $L, 1 \leq L \leq N$, and let $P_{v}=P_{v}(A)$ be projection onto $A$. For each vector $\xi$ in $\left(k_{v}\right)^{N}$ we have

(i) if $v \mid \infty$ then

(ii) if $v \nmid \infty$ then

$$
\|\boldsymbol{\xi}\|_{v}^{2}=\left\|P_{v} \boldsymbol{\xi}\right\|_{v}^{2}+\left\|\left(1_{N}-P_{v}\right) \boldsymbol{\xi}\right\|_{v}^{2}
$$

$$
|\boldsymbol{\xi}|_{v}=\max \left\{\left|P_{v} \boldsymbol{\xi}\right|_{v},\left|\left(1_{N}-P_{v}\right) \boldsymbol{\xi}\right|_{v}\right\} .
$$

PROOF. If $v \mid \infty$ then $P_{v} \xi$ and $\left(1_{N}-P_{v}\right) \xi$ are orthogonal with respect to the usual Hermitian inner product. Thus (i) is the familiar Pythagorean identity.

If $v \nmid \infty$ let $\left\{\mathbf{a}_{1}, \ldots, \mathbf{a}_{L}\right\}$ be a basis for $A$ and let $A=\left(\mathbf{a}_{1} \cdots \mathbf{a}_{L}\right)$ be the corresponding $N \times L$ matrix. By permuting the rows of $A$, if necessary, we may assume without loss of generality that the subset $J=J_{v} \subseteq\{1,2, \ldots, N\},|J|=L$, which satisfies (4.2), is in fact $J=\{1,2,3, \ldots, L\}$. If we write $W=A\left({ }_{J} A\right)^{-1}$ then ${ }_{J} W$ is $1_{L}$ and, using (4.2), we find that $\left|\operatorname{det}_{I} W\right|_{v} \leq 1$ holds for every $L \times L$ submatrix ${ }_{I} W$. In particular, if we form a submatrix ${ }_{I} W$ with $I=\{1,2, \ldots, l-1, l+1, \ldots, L$ and $L+j\}$, where $1 \leq j \leq N-L$, we obtain

$$
\left|\operatorname{det}_{I} W\right|_{v}=\left|w_{L+j, l}\right|_{v} \leq 1 \text {. }
$$

Thus every entry in the matrix $W$ has $v$-adic absolute value less than or equal to one. Therefore we must have

$$
\left|P_{v} \boldsymbol{\xi}\right|_{v}=\left|A\left({ }_{J} A\right)^{-1}{ }_{J}\left(1_{N}\right) \boldsymbol{\xi}\right|_{v}=\left|W\left({ }_{J} \boldsymbol{\xi}\right)\right|_{v} \leq|\boldsymbol{\xi}|_{v} .
$$

In a similar manner we can show that

$$
\left|\left(1_{N}-P_{v}\right) \boldsymbol{\xi}\right|_{v} \leq|\boldsymbol{\xi}|_{v} .
$$

Now by the ultrametric inequality,

$$
|\boldsymbol{\xi}|_{v}=\left|P_{v} \boldsymbol{\xi}+\left(1_{N}-P_{v}\right) \boldsymbol{\xi}\right|_{v} \leq \max \left\{\left|P_{v} \boldsymbol{\xi}\right|_{v},\left|\left(1_{n}-P_{v}\right) \boldsymbol{\xi}\right|_{v}\right\}
$$

Of course the identity in (ii) follows from (4.17), (4.18) and (4.19). 
LEMMA 8. Let $A \subseteq\left(k_{v}\right)^{N}$ and $P_{v}=P_{v}(\AA)$ be as in Lemma 7, and define $Q_{v}=Q_{v}(A)$ by $Q_{v}=\frac{1}{2}\left(1_{N}+P_{v}\right)$. For each vector $\boldsymbol{\xi}$ in $\left(k_{v}\right)^{N}$ we have

(i) if $v \mid \infty$ then $|\boldsymbol{\xi}|_{v} \leq 2^{d_{v} / d}\left|Q_{v} \boldsymbol{\xi}\right|_{v} \leq 2^{d_{v} / d}|\boldsymbol{\xi}|_{v}$,

(ii) if $v \mid 2$ then $|\boldsymbol{\xi}|_{v} \leq\left|Q_{v} \boldsymbol{\xi}\right|_{v} \leq 2^{d_{v} / d}|\boldsymbol{\xi}|_{v}$,

(iii) if $v \mid p$, where $p$ is an odd prime, then $|\boldsymbol{\xi}|_{v}=\left|Q_{v} \boldsymbol{\xi}\right|_{v}$.

PROOF. Suppose that $v \mid \infty$ and write $Q_{v}=P_{v}+\frac{1}{2}\left(1_{N}-P_{v}\right)$. Using (i) of Lemma 7 we have

$$
\begin{aligned}
\|\boldsymbol{\xi}\|_{v}^{2} & =\left\|P_{v} \boldsymbol{\xi}\right\|_{v}^{2}+\left\|\left(1_{N}-P_{v}\right) \boldsymbol{\xi}\right\|_{v}^{2} \\
& \leq 4\left\{\left\|P_{v} \boldsymbol{\xi}\right\|_{v}^{2}+\left\|\frac{1}{2}\left(1_{N}-P_{v}\right) \boldsymbol{\xi}\right\|_{v}^{2}\right\}=4\left\|Q_{v} \boldsymbol{\xi}\right\|_{v}^{2} \\
& \leq 4\left\{\left\|P_{v} \boldsymbol{\xi}\right\|_{v}^{2}+\left\|\left(1_{N}-P_{v}\right) \boldsymbol{\xi}\right\|_{v}^{2}\right\}=4\|\boldsymbol{\xi}\|_{v}^{2},
\end{aligned}
$$

and case (i) of the present lemma clearly follows from this. If $v \nmid \infty$ the proof is similar using (ii) of Lemma 7. We also take into account that $|2|_{v}=2^{-d_{v} / d}$ if $v \mid 2$ while $|2|_{v}=1$ if $v \mid p$ with $p$ an odd prime.

LEMMA 9. Let $A \subseteq\left(k_{v}\right)^{N}, P_{v}=P_{v}(\mathcal{A})$ and $Q_{v}=Q_{v}(\mathcal{A})$ be as in Lemma 8 . Suppose that $X$ is an $N \times M$ matrix over $k_{v}$ with $1 \leq \operatorname{rank}(X)=M \leq N$.

(i) If $v \mid \infty$ then

$$
H_{v}(X) \leq 2^{d_{v} M / d} H_{v}\left(Q_{v} X\right) \leq 2^{d_{v} M / d} H_{v}(X) .
$$

(ii) If $v \mid 2$ then

$$
H_{v}(X) \leq H_{v}\left(Q_{v} X\right) \leq 2^{d_{v} M / d} H_{v}(X) .
$$

(iii) If $v \mid p$, where $p$ is an odd prime, then $H_{v}(X)=H_{v}\left(Q_{v} X\right)$.

PROOF. The matrix $Q_{v}$ is clearly nonsingular. In fact, we have $Q_{v}^{-1}=(2) 1_{N}-$ $P_{v}$. Thus the columns of $Y=Q_{v} X$ span an $M$ dimensional subspace of $\left(k_{v}\right)^{N}$. By Lemma 6 , there is a nonsingular $M \times M$ matrix $U$ such that $Y U=\left(\mathbf{y}_{1}^{\prime} \mathbf{y}_{2}^{\prime} \cdots \mathbf{y}_{M}^{\prime}\right)$ satisfies

$$
H_{v}(Y U)=\prod_{m=1}^{M} H_{v}\left(\mathbf{y}_{m}^{\prime}\right)
$$

Suppose that $v \mid \infty$. Then

$$
H_{v}(X)=|\operatorname{det} U|_{v}^{-1} H_{v}\left(Q_{v}^{-1} Y U\right) \leq|\operatorname{det} U|_{v}^{-1} \prod_{m=1}^{M} H_{v}\left(Q_{v}^{-1} \mathbf{y}_{m}^{\prime}\right)
$$

by (2.3). Using (i) of Lemma 8 this is

$$
\leq|\operatorname{det} U|_{v}^{-1} 2^{d_{v} M / d} \prod_{m=1}^{M} H_{v}\left(\mathbf{y}_{m}^{\prime}\right) .
$$

Finally, we apply (4.20) to conclude that

$$
H_{v}(X) \leq|\operatorname{det} U|_{v}^{-1} 2^{d_{v} M / d} H_{v}(Y U)=2^{d_{v} M / d} H_{v}\left(Q_{v} X\right) .
$$

All the remaining cases can be proved in a similar manner using Lemma 6 and the other inequalities in Lemma 8. 
5. Enlarging subspaces. Let $\mathcal{X}$ and $Z$ be subspaces of $(k)^{N}$ such that $\chi \subseteq$ $Z \subseteq(k)^{N}$ and

$$
1 \leq \operatorname{dim}(X)=L<\operatorname{dim}(Z)=M \leq N .
$$

We assume that $\left\{\mathbf{x}_{1}, \mathbf{x}_{2}, \ldots, \mathbf{x}_{L}\right\}$ is a basis for $\mathcal{X}$ over $k$ and $\left\{\mathbf{x}_{1}, \ldots \mathbf{x}_{L}, \mathbf{y}_{1}, \mathbf{y}_{2}, \ldots\right.$, $\left.\mathbf{y}_{M-L}\right\}$ is a basis for $Z$ over $k$. We write

$$
X=\left(\mathbf{x}_{1} \mathbf{x}_{2} \cdots \mathbf{x}_{L}\right), \quad Y=\left(\mathbf{y}_{1} \mathbf{y}_{2} \cdots \mathbf{y}_{M-L}\right), \quad \text { and } \quad Z=\left(\begin{array}{ll}
X & Y
\end{array}\right)
$$

for the corresponding $N \times L, N \times(M-L)$ and $N \times M$ matrices over $k$.

At each place $v$ of $k$ we write $X_{v}$ and $Z_{v}$ for the completions of $X$ and $Z$. Thus $X_{v} \subseteq Z_{v} \subseteq\left(k_{v}\right)^{N}$ are subspaces spanned by $\left\{\mathbf{x}_{1}, \ldots, \mathbf{x}_{L}\right\}$ and $\left\{\mathbf{x}_{1}, \ldots, \mathbf{x}_{L}, \mathbf{y}_{1}, \ldots\right.$, $\left.\mathbf{y}_{M-L}\right\}$ over $k_{v}$, respectively. At each place $v$ we use the matrix $X$ to define projection onto $X_{v}$. Now we write $P_{v}=P_{v}(X)=P_{v}\left(X_{v}\right)$ for the $N \times N$ matrix defined by (4.1) or (4.3).

Suppose that $H(X)$ is large compared with $H(Z)$. In this section we wish to show that an intermediate subspace $\mathcal{Y}$ can be constructed such that $\chi \subseteq \mathcal{Y} \subseteq Z$, the dimension of $\mathcal{Y}$ is not too large and $H(\mathcal{Y})$ is smaller that $H(X)$. For our eventual application we require only the case in which $y$ has dimension $L+1$. However, there are no additional difficulties in proving the following more general result.

THEOREM 10. There exist $M-L$ linearly independent vectors $\mathbf{u}_{1}, \mathbf{u}_{2}, \ldots$, $\mathbf{u}_{M-L}$ in $k^{M-L}$ such that

$$
\prod_{l=1}^{M-L}\left\{\prod_{v} H_{v}\left\{\left(1_{N}-P_{v}\right) Y \mathbf{u}_{l}\right\}\right\} \leq\left\{c_{k}(M-L)\right\}^{M-L} H(Z) H(X)^{-1} .
$$

Proof. If $v \mid \infty$ we define

$$
S_{v}=\left\{\mathbf{u} \in\left(k_{v}\right)^{M-L}:\left\|\left(1_{N}-P_{v}\right) Y \mathbf{u}\right\|_{v}<r_{v}(M-L)\right\} .
$$

The set $S_{v}$ is nonempty, open, convex and symmetric. Our choice of $r_{v}(M-L)$ is such that

$$
\beta_{v}^{M-L}\left(\left\{\mathbf{u} \in\left(k_{v}\right)^{M-L}:\|\mathbf{u}\|_{v}<r_{v}(M-L)\right\}\right)=1 .
$$

Since \|\|$_{v}$ is the usual Hermitian norm on vectors, it follows easily that

$$
\beta_{v}^{M-L}\left(S_{v}\right)=\left\|\operatorname{det}\left\{\left(1_{N}-P_{v}\right) Y\right\}^{*}\left\{\left(1_{N}-P_{v}\right) Y\right\}\right\|_{v}^{-d_{v} / 2}=H_{v}\left\{\left(1_{N}-P_{v}\right) Y\right\}^{-d}
$$

If $v \nmid \infty$ we define

$$
S_{v}=\left\{\mathbf{u} \in\left(k_{v}\right)^{M-L}:\left\|\left(1_{N}-P_{v}\right) Y \mathbf{u}\right\|_{v} \leq 1\right\} .
$$

In this case the set $S_{v}$ is a compact and open $O_{v}$-module in $\left(k_{v}\right)^{M-L}$. The Haar measure of $S_{v}$ can be found by using the $v$-adic cube slicing identity which is (4.8) and (4.9) of [2]. By that result we have

$$
\beta_{v}^{M-L}\left(S_{v}\right)=H_{v}\left\{\left(1_{N}-P_{v}\right) Y\right\}^{-d}\left|D_{v}\right|_{v}^{d(M-L) / 2} .
$$

Also, for almost all finite places $v$ the entries in the matrix $\left(1_{N}-P_{v}\right) Y$ have $v$-adic absolute value less than or equal to one. It follows that $\left(O_{v}\right)^{M-L} \subseteq S_{v}$ for almost all $v$. On the other hand, we have $H_{v}(Z)=H_{v}(X)=1$ for almost all finite $v$ and

$$
H_{v}\left\{\left(1_{N}-P_{v}\right) Y\right\}=H_{v}(Z) H_{v}(X)^{-1}
$$


by Lemma 4 . It follows from (5.2) that

$$
\beta_{v}^{M-L}\left(S_{v}\right)=\left|D_{v}\right|_{v}^{d(M-L) / 2}=\beta_{v}^{M-L}\left(\left(O_{v}\right)^{M-L}\right)
$$

at almost all finite $v$ and hence $S_{v}=\left(O_{v}\right)^{M-L}$ at almost all finite $v$.

Let $S=\prod_{v} S_{v}$, so that by our previous remarks $S$ is a subset of the product of adèle spaces $\left(k_{\mathbf{A}}\right)^{M-L}$. We then define $\lambda_{l}, l=1,2, \ldots, M-L$, to be the $l$ th successive minima associated with $S$. That is,

$$
\lambda_{l}=\inf \left\{\lambda>0:(\lambda S) \cap(k)^{M-L} \text { contains } l \text { linearly independent vectors }\right\} .
$$

The set $S$ has a compact closure in $\left(k_{\mathbf{A}}\right)^{M-L}$ so that its adèlic Haar measure is

$$
V(S)=\prod_{v} \beta_{v}^{M-L}\left(S_{v}\right)
$$

Applying (5.1), (5.2) and Lemma 4 we have

$$
\begin{aligned}
V(S) & =\left(\prod_{v} H_{v}\left\{\left(1_{N}-P_{v}\right) Y\right\}\right)^{-d}\left(\prod_{v+\infty}\left|D_{v}\right|^{d(M-L) / 2}\right) \\
& =H(Z)^{-d} H(X)^{d}\left|\Delta_{k}\right|^{-(M-L) / 2} .
\end{aligned}
$$

Now the successive minima $\lambda_{1}, \ldots, \lambda_{M-L}$ satisfy $\left(\lambda_{1} \lambda_{2} \cdots \lambda_{M-L}\right)^{d} V(S) \leq 2^{d(M-L)}$ (this is Theorem 3 of [2]) and therefore

$$
\left(\lambda_{1} \lambda_{2} \cdots \lambda_{M-L}\right) \leq\left(2\left|\Delta_{k}\right|^{1 / 2 d}\right)^{M-L} H(Z) H(X)^{-1} .
$$

Next we suppose that $\mathbf{u} \neq \mathbf{0}$ is a vector in $(\lambda S) \cap(k)^{M-L}$ for some $\lambda>0$. We have

$$
\begin{cases}\left\|\left(1_{N}-P_{v}\right) Y \mathbf{u}\right\|_{v}<\lambda r_{v}(M-L) & \text { if } v \mid \infty, \\ \left|\left(1_{N}-P_{v}\right) Y \mathbf{u}\right|_{v} \leq 1 & \text { if } v \nmid \infty,\end{cases}
$$

and therefore

$$
\prod_{v} H_{v}\left\{\left(1_{N}-P_{v}\right) Y \mathbf{u}\right\}<\lambda \prod_{v+\infty}\left\{r_{v}(M-L)\right\}^{d_{v} / d}
$$

In particular, let $\left\{\mathbf{u}_{1}, \mathbf{u}_{2}, \ldots, \mathbf{u}_{M-L}\right\}$ be $k$-linearly independent vectors associated with the successive mimina $\lambda_{1} \leq \lambda_{2} \leq \cdots \leq \lambda_{M-L}$. We may apply (5.3) to each $\mathbf{u}_{l}$ and $\lambda>\lambda_{l}$. It follows that

$$
\begin{aligned}
& \prod_{l=1}^{M-L}\left\{\prod_{v} H_{v}\left\{\left(1_{N}-P_{v}\right) Y \mathbf{u}_{l}\right\}\right\} \leq\left\{\prod_{l=1}^{M-L} \lambda_{l}\right\}\left\{\prod_{v \mid \infty}\left(r_{v}(M-L)\right)^{d_{v} / d}\right\}^{M-L} \\
& \leq\left\{2\left|\Delta_{k}\right|^{1 / 2 d} \prod_{v \mid \infty}\left(r_{v}(M-L)\right)^{d_{v} / d}\right\}^{M-L} H(Z) H(\mathcal{X})^{-1} \\
& =\left\{c_{k}(M-L)\right\}^{M-L} H(Z) H(X)^{-1} \text {. }
\end{aligned}
$$

COROLLARY 11. There exists a vector $\boldsymbol{\xi} \neq 0$ in $Z$ such that

(i) the subspace $\mathcal{Y}$ which is spanned by $\left\{\mathbf{x}_{1}, \ldots, \mathbf{x}_{L}, \boldsymbol{\xi}\right\}$ over $k$ has dimension $L+1$. 
(ii) the vectors $\left(1_{N}-P_{v}\right) \xi$ satisfy

$$
\prod_{v} H_{v}\left\{\left(1_{N}-P_{v}\right) \xi\right\} \leq c_{k}(M-L) H(Z)^{1 /(M-L)} H(X)^{-1 /(M-L)},
$$

(iii) the subspace $\mathcal{Y}$ satisfies

$$
H(\mathcal{Y}) \leq c_{k}(M-L) H(Z)^{1 /(M-L)} H(\mathcal{X})^{1-1 /(M-L)} .
$$

PROOF. We may assume that $\mathbf{u}_{1}, \mathbf{u}_{2}, \ldots, \mathbf{u}_{M-L}$ in the theorem are arranged so that $\prod_{v} H_{v}\left\{\left(1_{N}-P_{v}\right) Y \mathbf{u}_{l}\right\}$ is increasing with $l$. Then we set $\boldsymbol{\xi}=Y \mathbf{u}_{1}$. Conditions (i) and (ii) follow immediately. Using Lemma 4 we have

$$
\begin{aligned}
H(\mathcal{Y}) & =\prod_{v} H_{v}\left\{\left(\begin{array}{ll}
X & \xi
\end{array}\right)\right\}=\prod_{v} H_{v}\left\{\left(\begin{array}{ll}
X & \left(1_{N}-P_{v}\right) \xi
\end{array}\right)\right\} \\
& =H(X) \prod_{v} H_{v}\left\{\left(1_{N}-P_{v}\right) \xi\right\} \leq c_{k}(M-L) H(Z)^{1 /(M-L)} H(\mathcal{X})^{1-1 /(M-L)} .
\end{aligned}
$$

6. Proof of Theorem 1. Let $F, \Phi, L$ and $Z$ be as defined in $\S 3$. Then $Z^{(0)}$ contains a subspace of dimension $L$. Among all $L$-dimensional subspaces contained in $Z^{(0)}$, let $\mathcal{X}$ have minimal height. Since the set of all $L$-dimensional subspaces of $k^{N}$ having height less than or equal to a constant is a finite set, such an $\mathcal{X}$ clearly exists. Let $\left\{\mathbf{x}_{1}, \mathbf{x}_{2}, \ldots, \mathbf{x}_{L}\right\}$ be a basis for $\mathcal{X}$ and let $X=\left(\mathbf{x}_{1} \mathbf{x}_{2} \cdots \mathbf{x}_{L}\right)$ be the corresponding $N \times L$ matrix. We suppose that $\mathcal{Y}$ is a subspace of $Z$, spanned by $\left\{\mathbf{x}_{1}, \mathbf{x}_{2}, \ldots, \mathbf{x}_{L}, \boldsymbol{\xi}\right\}$ over $k$, which satisfies conditions (i), (ii) and (iii) of Corollary 11. Let $\mathbf{y} \in \mathcal{Y}$ so that

$$
\mathbf{y}=\sum_{l=1}^{L} \alpha_{l} \mathbf{x}_{l}+\beta \boldsymbol{\xi}
$$

with $\alpha_{l} \in k$ and $\beta \in k$. It follows that

$$
F(\mathbf{y})=2 F\left(\sum_{l=1}^{L} \alpha_{l} \mathbf{x}_{l}, \beta \boldsymbol{\xi}\right)+F(\beta \boldsymbol{\xi})=2 \beta\left\{\sum_{l=1}^{L} \alpha_{l} F\left(\mathbf{x}_{l}, \boldsymbol{\xi}\right)+\frac{1}{2} \beta F(\boldsymbol{\xi})\right\} .
$$

From the definition of $L$ we know that $F$ cannot vanish on all vectors in $\mathcal{Y}$. Thus there exists a vector $\mathbf{z} \in \mathcal{Y}$ such that $F(\mathbf{z}) \neq 0$. Let

$$
\mathbf{z}=\sum_{l=1}^{L} \gamma_{l} \mathbf{x}_{l}+\delta \boldsymbol{\xi}
$$

with $\gamma_{l} \in k$ and $\delta \in k$. Clearly $\delta \neq 0$, for otherwise $\mathbf{z}$ would be in $\mathcal{X}$ contrary to the fact that $F(\mathbf{z}) \neq 0$. Expanding $F(\mathbf{z})$ as in (6.1), we see that

$$
F\left(\mathbf{x}_{l}, \boldsymbol{\xi}\right) \neq 0 \quad \text { for some } l, 1 \leq l \leq L, \quad \text { or } \quad F(\boldsymbol{\xi}) \neq 0 .
$$

Next we define a subspace $X^{\prime} \subseteq \mathcal{Y}$ by

$$
\chi^{\prime}=\left\{\sum_{l=1}^{L} \alpha_{l} \mathbf{x}_{l}+\beta \boldsymbol{\xi}: \alpha_{l} \in k, \beta \in k, \text { and } \sum_{l=1}^{L} \alpha_{l} F\left(\mathbf{x}_{l}, \boldsymbol{\xi}\right)+\frac{1}{2} \beta F(\boldsymbol{\xi})=0\right\} .
$$

Because of (6.2), the linear equation satisfied by $\alpha_{1}, \ldots, \alpha_{L}, \beta$ in the definition of $X^{\prime}$ is nontrivial. Thus $X^{\prime}$ has dimension $L$. It follows from the expansion (6.1) that 
$F$ vanishes identically on $\chi^{\prime}$. In view of the extremal property satisfied by $\chi$, we must have

$$
H(\chi) \leq H\left(\chi^{\prime}\right)
$$

We now proceed to give an upper bound for the height of $\chi^{\prime}$. Let $\left\{\mathbf{x}_{1}^{\prime}, \mathbf{x}_{2}^{\prime}, \ldots, \mathbf{x}_{L}^{\prime}\right\}$ be a basis for $X^{\prime}$ over $k$ and let $X^{\prime}=\left(\mathbf{x}_{1}^{\prime} \mathbf{x}_{2}^{\prime} \cdots \mathbf{x}_{L}^{\prime}\right\}$ be the corresponding $N \times L$ matrix. Obviously $\mathbf{z} \notin X^{\prime}$ so that $\left\{\mathbf{x}_{1}^{\prime}, \mathbf{x}_{2}^{\prime}, \ldots, \mathbf{x}_{L}^{\prime}, \mathbf{z}\right\}$ is a basis for $\mathcal{Y}$ and $H(\mathcal{Y})=$ $H\left(\begin{array}{ll}X^{\prime} & \mathbf{z}\end{array}\right)$.

At each place $v$ of $k$ let $P_{v}=P_{v}(X)=P_{v}\left(X_{v}\right)$ and $Q_{v}=\frac{1}{2}\left(1_{N}+P_{v}\right)$. We claim that

$$
F\left(Q_{v} \mathbf{x}_{l}^{\prime},\left(1_{N}-P_{v}\right) \boldsymbol{\xi}\right)=0 \text { for } 1 \leq l \leq L
$$

and

$$
F\left(Q_{v} \mathbf{z},\left(1_{N}-P_{v}\right) \boldsymbol{\xi}\right)=(2 \delta)^{-1} F(\mathbf{z}) \neq 0 .
$$

To verify (6.4) write

$$
\mathbf{x}_{l}^{\prime}=\sum_{m=1}^{L} \alpha_{m l} \mathbf{x}_{m}+\beta_{l} \boldsymbol{\xi}
$$

We have

$$
Q_{v} \mathbf{x}_{l}^{\prime}=\sum_{m=1}^{L} \alpha_{m l} \mathbf{x}_{m}+\frac{1}{2} \beta_{l}\left(1_{N}+P_{v}\right) \boldsymbol{\xi}
$$

and therefore

$$
\begin{aligned}
F\left(Q_{v} \mathbf{x}_{l}^{\prime},\left(1_{N}-P_{v}\right) \boldsymbol{\xi}\right) & =F\left(\sum_{m=1}^{L} \alpha_{m l} \mathbf{x}_{m}, \boldsymbol{\xi}\right)+\frac{1}{2} \beta_{l} F\left(\left(1_{N}+P_{v}\right) \boldsymbol{\xi},\left(1_{N}-P_{v}\right) \boldsymbol{\xi}\right) \\
& =\sum_{m=1}^{L} \alpha_{m l} F\left(\mathbf{x}_{m}, \boldsymbol{\xi}\right)+\frac{1}{2} \beta_{l} F(\boldsymbol{\xi}) .
\end{aligned}
$$

In fact this last expression is zero since $\mathbf{x}_{l}^{\prime}$ is in $\chi^{\prime}$. The identity (6.5) follows in a similar manner. We find that

$$
\begin{aligned}
F\left(Q_{v} \mathbf{z},\left(1_{N}-P_{v}\right) \boldsymbol{\xi}\right) & =F\left(\sum_{m=1}^{L} \gamma_{m} \mathbf{x}_{m}, \boldsymbol{\xi}\right)+\frac{1}{2} \delta F(\boldsymbol{\xi}) \\
& =(2 \delta)^{-1} F(\mathbf{z}),
\end{aligned}
$$

where we have used (6.1), with $\mathbf{y}$ replaced by $\mathbf{z}$, at the last step.

At each place $v$ of $k$ we define $\Psi_{v}$ by

$$
\Psi_{v}=\left\{\left(1_{N}-P_{v}\right) \xi\right\}^{T} \Phi
$$

so that $\Psi_{v}$ is a $1 \times N$ matrix over $k_{v}$. Using the Cauchy-Schwarz inequality or the ultrametric inequality, we easily conclude that

$$
H_{v}\left(\Psi_{v}\right) \leq H_{v}(\Phi) H_{v}\left\{\left(1_{N}-P_{v}\right) \xi\right\} .
$$

The matrix $\Psi_{v}$ can be used to rewrite (6.4) and (6.5) as

$$
\Psi_{v} Q_{v} X^{\prime}=0 \text { and } \Psi_{v} Q_{v} \mathbf{z}=(2 \delta)^{-1} F(\mathbf{z}) .
$$


Thus $\Psi_{v}$ satisfies the conditions of Lemma 5 with $M=L+1$. Applying (4.13) we obtain the inequality

$$
\left|(2 \delta)^{-1} F(\mathbf{z})\right|_{v} H_{v}\left(Q_{v} X^{\prime}\right) \leq H_{v}\left(\Psi_{v}\right) H_{v}\left\{\left(Q_{v} X^{\prime} \quad Q_{v} \mathbf{z}\right)\right\} .
$$

At this point we may appeal to Lemma 9 which allow us to remove the matrix $Q_{v}$ from both sides of (6.7) while compensating with additional factors of the form $2^{-d_{v} L / d}$ if $v \mid \infty$ and $2^{d_{v}(L+1) / d}$ if $v \mid 2$. We also use the product formula and find that

$$
\begin{aligned}
2^{-L} \prod_{v} H_{v}\left(X^{\prime}\right) & =2^{-L} H\left(X^{\prime}\right) \leq 2^{L+1} \prod_{v}\left\{H_{v}\left(\Psi_{v}\right) H_{v}\left(X^{\prime} \quad \mathbf{z}\right)\right\} \\
& =2^{L+1}\left(\prod_{v} H_{v}\left(\Psi_{v}\right)\right) H(\mathcal{Y}) .
\end{aligned}
$$

Of course we have

$$
\begin{aligned}
\prod_{v} H_{v}\left(\Psi_{v}\right) & \leq \mathcal{H}(\Phi) \prod_{v} H_{v}\left\{\left(1_{N}-P_{v}\right) \xi\right\} \\
& \leq c_{k}(M-L) H(\Phi) H(Z)^{1 /(M-L)} H(\mathcal{H})^{-1 /(M-L)}
\end{aligned}
$$

from (6.6) and part (ii) of Corollary 11. Finally, we combine (6.3), (6.8), (6.9) and part (iii) of Corollary 11. This leads to the estimate

$$
H(X) \leq H\left(\chi^{\prime}\right) \leq 2^{2 L+1} c_{k}(M-L)^{2} \not(\Phi) H(Z)^{2 /(M-L)} H(X)^{1-2 /(M-L)} ;
$$

and hence to

$$
H(\mathcal{X}) \leq\left\{2^{2 L+1} c_{k}(M-L)^{2} \mathcal{H}(\Phi)\right\}^{(M-L) / 2} H(Z) .
$$

7. Proof of the corollaries. Suppose that $\chi \subseteq Z^{(0)}$ is the $L$-dimensional subspace satisfying (3.1) and $X$ is the $N \times L$ matrix having columns which span $\mathcal{X}$ over $k$. By Theorem 8 in [2] there exist $L$ linearly independent vectors $\mathbf{u}_{1}, \mathbf{u}_{2}, \ldots, \mathbf{u}_{L}$ in $k^{L}$ such that $X \mathbf{u}_{l} \in\left(O_{k}\right)^{N}$ and

$$
\prod_{l=1}^{L} h\left(X \mathbf{u}_{l}\right) \leq c_{k}(1)^{L} H(X) .
$$

Corollary 2 follows by setting $\varsigma_{l}=X \mathbf{u}_{l}$ and using (3.1).

To establish Corollary 3 let $M=N-K$ and define $Z$ to be the $M$ dimensional subspace $Z=\left\{\mathrm{z} \in k^{N}: A \mathrm{z}=0\right\}$. By the duality principle (2.6) we have $H(Z)=H(A)$. Thus (3.4) follows immediately from Theorem 1 and (3.5) is equally straighforward from Corollary 2.

\section{REFERENCES}

1. B. J. Birch and H. Davenport, Quadratic equations in several variables, Proc. Cambridge Philos. Soc. 54 (1958), 135-138.

2. E. Bombieri and J. Vaaler, On Siegel's lemma, Invent. Math. 73 (1983), 11-32.

3. J. W. S. Cassels, Bounds for the least solution of homogeneous quadratic equations, Proc. Cambridge Philos. Soc. 51 (1955), 262-264.

4. J. H. H. Chalk, Linearly independent zeros of quadratic forms over number fields, Monatsh. Math. 90 (1980), 13-25.

5. P. Gordan, Über den grössten gemeinsamen Factor, Math. Ann. 7 (1873), 443-448. 
6. W. V. D. Hodge and D. Pedoe, Methods of algebraic geometry, vol. 1, Cambridge Univ. Press, 1968.

7. S. Raghavan, Bounds of minimal solutions of diophantine equations, Nachr. Akad. Wiss. Gottingen, Math. Phys. Kl. 9 (1975), 109-114.

8. H. P. Schlickewei, Kleine Nullstellen homogener quadratischer Gleichungen, Monatsh. Math. 100 (1985), 35-45.

Department of Mathematics, University of Texas, Austin, Texas 78712 